\title{
Human Immunodeficiency Virus-infected Macrophages Produce Soluble Factors that Cause Histological and Neurochemical Alterations in Cultured Human Brains
}

Lynn Pulliam, * Brian G. Herndier," Norina M. Tang," and Michael S. McGrath"

*Department of Laboratory Medicine, San Francisco Veterans Administration Medical Center and University of California, San Francisco, California 94121; ' Department of Pathology, San Francisco General Hospital and University of California, San Francisco, California 94110; and the ${ }^{\S}$ AIDS Program, San Francisco General Hospital and University of California, San Francisco, California 94110

\begin{abstract}
We wanted to establish an in vitro human model for AIDS-associated dementia and pursue the hypothesis that this disease process may be a result of soluble factors produced by HIV-infected macrophages. Human brain aggregates were prepared from nine different brain specimens, and were treated with supernatants from in vitro HIV-infected macrophages (SI), uninfected macrophages (SU), infected T cells, or macrophage-conditioned media from four AIDS patients.

Seven of nine treated brains exposed to SI showed peripheral rarefaction after $1 \mathrm{wk}$ of incubation that by ultrastructural analysis showed cytoplasmic vacuolation. Aggregates from two of three brain cultures treated with SI for 3 wk became smaller, an $\sim 50 \%$ decrease in size. The degree of apparent toxicity in brains exposed to patient-derived macrophage supernatants paralleled the proportion of macrophages found to be expressing HIV p24. Ultrastructural abnormalities were not observed in brains treated with supernatants from HIV-infected T cells, uninfected macrophages, or LPS-activated macrophages. Levels of five neurotransmitter amino acids were decreased in comparison to the structural amino acid leucine. These findings suggest that HIV-infected macrophages, infected both in vitro as well as derived from AIDS patients' peripheral blood, produce factors that cause reproducible histochemical, ultrastructural, and functional abnormalities in human brain aggregates. (J. Clin. Invest. 1991. 87:503-512.) Key words: AIDS• dementia $\bullet$ cyclic nucleotide phosphohydrolase $\bullet$ aggregate $\bullet$ neuropathology
\end{abstract}

\section{Introduction}

Neurological involvement, unrelated to tumors or opportunistic infections, frequently accompanies AIDS and has been referred to as the AIDS dementia complex. Clinically, AIDS dementia presents with cognitive impairment usually followed by motor abnormalities (1). Progression of this dementia can be mild or fulminant and has been shown to be variable among AIDS patients. In most cases of AIDS dementia, computed tomography $(\mathrm{CT})^{1}$ scanning and magnetic resonance imaging

Address correspondence to Dr. Lynn Pulliam, Veterans Administration Medical Center, 113A, 4150 Clement St., San Francisco, CA 94121.

Received for publication 19 October 1989 and in revised form 2 October 1990

1. Abbreviations used in this paper: CNP, cyclic neocleotide phosphohydrolase; CT, computed tomography; EAA, excitatory amino acids; LDH, lactate dehydrogenase; MOI, multiplicity of infection; MRI, magnetic resonance imaging; NAA, neurotransmitter amino acids; SI, infected supernatant; SU, uninfected supernatant; TNF, tumor necrosis factor.

The Journal of Clinical Investigation, Inc.

Volume 87, February 1991, 503-512
(MRI) analysis reveal cerebral atrophy (1). Neuropathologic findings in brains of patients with AIDS dementia complex include focal rarefaction or spongiosis, vacuolation, and microglial nodule and multinucleated giant cell formation (2). Evidence to suggest direct HIV infection of neural cells has been reported. HIV DNA and RNA have been detected in brains of AIDS patients and infectious HIV has been isolated from brain tissue and cerebrospinal fluid (3-6). These data coupled with the neurologic symptoms experienced by $60 \%$ of patients with AIDS (7) strongly suggest that HIV is neurotropic.

Productive HIV infection of primary cultured neural cells $(8,9)$ has been difficult, and in the absence of cytotoxic infection of neurons, astrocytes, and oligodendrocytes, the pathophysiology of central nervous system (CNS) dysfunction in AIDS may not be related to direct cellular infection. This evidence supports the notion that an indirect mechanism may be responsible for the neurological damage seen in AIDS dementia. Three possible explanations have been suggested. First, products of virus replication may cause damage to surrounding cells. The envelope glycoprotein (gp120) of HIV has been shown to cause neuronal cell death in hippocampal cultures of fetal mice (10) and stimulate monocyte function resulting in damaging physiological metabolites (11). Secondly, HIV gene products may interfere with factors necessary for neural cell function. The HIV gp1 20 has been shown to inhibit the function of neuroleukin, a neurotropic factor that enhances neuronal survival in culture (12). Finally, HIV-infected cells may produce factors that alter neural cell function.

In AIDS patients, the cell type that appears to be an in vivo reservoir of HIV infection is the monocyte/macrophage (13, 14). In the brain, the CNS macrophage or microglial cell may also serve as a reservoir for HIV. Unlike the $\mathrm{CD} 4^{+}$lymphocyte, the macrophage appears to survive HIV infection. Recently, HIV-infected macrophages were found to produce a potent cytokine termed contra IL-1 that blocked IL-1-mediated T cell activation (15). The current studies were conducted to test the hypothesis that HIV-infected macrophages might produce soluble factors capable of altering normal brain structure and function in vitro.

\section{Methods}

Brain aggregate cultures. These experiments used a normal human brain aggregate culture system that contained all the cells of the central nervous system with accompanying myelin (16). This system allows for easy treatment and sampling of brain over time in culture. Human fetal brain tissue between 15 and $18 \mathrm{wk}$ gestation was obtained in accordance with the University of California, San Francisco Committee on Human Research and was gently dissociated through nylon screens to obtain single cells. Suspensions with $>50 \%$ viability as determined by trypan blue exclusion were counted on a hemocytometer, and $4 \times 10^{7}$ cells within $4 \mathrm{ml}$ DME supplemented with $0.6 \%$ dextrose, $50 \mu \mathrm{g} / \mathrm{ml}$ gentamicin, and $10 \%$ FCS were distributed into $25-\mathrm{ml}$ DeLong flasks 
(Bellco Biotechnology, Vineland, NJ). Aggregate cultures were constantly rotated throughout the experiments and incubated at $37^{\circ} \mathrm{C}$ in an atmosphere of $10 \% \mathrm{CO}_{2}$. After 2-3 d, the aggregate cultures were transferred to $50-\mathrm{ml}$ DeLong flasks and $5 \mathrm{ml}$ of DME supplemented with $15 \%$ FCS was added to each flask. 5-ml of medium was exchanged every 2-3 d and replaced with fresh medium alone or with added treatments. After $\sim 10 \mathrm{~d}$ in culture and before each experiment, aggregates were sampled for histology. Brain aggregates contain all the cells of the central nervous system including neurons, astrocytes, oligodendrocytes, microglial cells, and rare ependymal cells and macrophages. Neurons were recognized immunocytochemically by antibodies to $\alpha$-tubulin or neuron-specific enolase, astrocytes by antibodies to glial fibrillary acidic protein, oligodendrocytes by antibodies to $\beta$ galactocerebroside, and antibodies to myelin basic protein (16). Electron microscopic ultrastructure analysis was used to confirm findings at the light microscopy level and to identify other cells present. Astrocytes and neurons each make up $>40 \%$ of the cells present in aggregates. Neurons are irregular in size; their nuclei are often bilobed and usually contain a prominent nucleolus. The cytoplasm contains endoplasmic reticulum, ribosomes, Golgi apparatus, mitochondria, and small liposomes. Astrocytes contain cytoplasmic fibrils with glycogen granules, mitochondria, and a large irregularly shaped nucleus. Oligodendrocytes represent $\sim 10 \%$ of cells present in aggregates and are identified by spherical or oval nuclei with dark irregular nucleoplasm. The surrounding perikaryon contain rough endoplasmic reticulum, mitochondria, and often electron dense bodies. Microglia are identified as cells smaller than astrocytes and oligodendrocytes with irregular nuclei containing dense nucleoplasm and surrounded by scant cytoplasm. Cells the size of astrocytes that have an oval nucleus with abundant dense chromatin and a cytoplasm containing phagocytized cell debris, myelin figures, and lipid droplets are considered macrophages. Rarely is a cell observed that is characterized by a large nucleus, scant cytoplasm, and cilia, which are consistent with an ependymal cell.

Cyclic nucleotide phosphohydrolase (CNP) levels were determined to quantify possible demyelination in the brain aggregate system. The enzyme occurs in high levels in myelin and oligodendrocytes; thus, it has been used as a biochemical marker for myelination/demyelination processes (17). Brain aggregates can remain in culture and maintain their cell surface markers without added growth factors for up to 2 mo (16).

Supernatant preparation and treatment. Supernatants were pools prepared from seven different normal macrophage donors. Macrophages were isolated from peripheral blood of normal blood donors as previously described (13) and one-half of the macrophages in suspension culture were infected at a multiplicity of infection (MOI) of 1 with HIV $_{\text {DV }}$ strain. Both infected (SI) and uninfected (SU) macrophage culture supernatants were changed weekly with cells seeded at a concentration of $\sim 2 \times 10^{5}$ cells $/ \mathrm{ml}$ in RPMI-1640 supplemented with $10 \%$ human serum. Infected and uninfected macrophages were maintained in Teflon culture vessels (Savillex Corp., Minnetonka, MN) in suspension culture. 2 wk after infection, up to $30 \%$ of the cells from each donor expressed HIV p24 as analyzed by flow cytometry using a p24-specific murine MAb (13). All supernatants were ultracentrifuged in an SW 28 rotor at $25,000 \mathrm{rpm}$ for $24 \mathrm{~h}\left(4^{\circ} \mathrm{C}\right)$ through a $25 \%$ sucrose cushion to insure complete removal of whole virus particles. Supernatants from HIV-infected macrophages were tested in a standard HIV titration system and found to be free of all infectious HIV. Pooled SI were found to contain $30,000 \mathrm{pg} \mathrm{HIV} \mathrm{p} 24 / \mathrm{ml}$, as measured by the Abbott HIV antigen capture assay. Tumor necrosis factor $\alpha$ (TNF- $\alpha$ ) and $\gamma$ interferon were not detected above background levels (assays performed courtesy of Dr. Arthur Ammann, Genentech Corp., So. San Francisco, CA).

For $T$ cell infections, a $T$ lymphoblastoid cell line (VB, also known as SUPT-1) was used. This is a CD4 $4^{+} \mathrm{T}$ lymphoma cell line (human $\mathrm{T}$ lymphotropic virus I-negative) that replicates HIV-1 to very high levels within 3-4 d of infection (14). Cells were inoculated with the isolate $\mathrm{HIV}_{\mathrm{Dv}}$ at an MOI of 0.005 . Cells were incubated for $60 \mathrm{~min}$ at $37^{\circ} \mathrm{C}$ and then washed to remove unbound virus. Cells were resuspended at
$1.0 \times 10^{5}$ cells $/ \mathrm{ml}$ in RPMI- 1640 supplemented with $10 \%$ FCS. Virusmediated cytopathic effects as well as HIV p24 levels peaked at $4 \mathrm{~d}$ after infection, at which time the supernatants were harvested. Pooled supernatants from HIV-infected VB cells were found to contain 350,000 $\mathrm{pg} / \mathrm{ml}$ of $\mathrm{HIV} \mathrm{p} 24 / \mathrm{ml}$ as measured by the Abbott HIV antigen capture assay after removal of infectious particles by ultracentrifugation.

Brain aggregates were incubated in media alone (described above), $20 \%$ uninfected macrophage supernatant (SU), 20\% HIV-infected macrophage supernatant (SI), or $20 \%$ HIV-infected T cell supernatant. Treatments were begun 12-13 d after initiation of cultures and aggregates were sampled weekly; media with or without $20 \%$ supernatant were exchanged three times weekly. Seven human brains (HB) were studied.

Patient HIV-infected macrophage supernatant preparation and treatment. $30 \mathrm{ml}$ of blood were obtained (unlinked) from four patients with AIDS in accordance with the University of California, San Francisco Committee on Human Research. The PBMC were separated through a Ficoll-Hypaque gradient, washed, and then were allowed to adhere to glass overnight as described for the uninfected macrophages above. After washing free any nonadherent cells, the adherent macrophages were scraped free, counted, and placed into teflon suspension culture at $5 \times 10^{5} \mathrm{cells} / \mathrm{ml}$ in the above described macrophage medium. After $5 \mathrm{~d}$ of cultivation, the cells were spun out of their culture supernatant, which was frozen at $-70^{\circ} \mathrm{C}$ until removal of virus particles by ultracentrifugation and subsequent use in brain aggregate culture systems. The harvested patient macrophages were then split into two aliquots after fixation, permeablization, and were stained with anti-HIV p24 MAb or irrelevant control antibodies as described above (13). Immunocytofluorometric analysis was then performed and the percent of cells that stained above background was enumerated for each specimen. A background fluorescence gate was arbitrarily set at $5 \% ; 5 \%$ of the isotype-matched control-stained macrophages fell within this gate. The percent $\mathrm{p} 24^{+}$cells was that percent above the $5 \%$ negative control with values of $1.5 \%$ and less considered no different than background. Supernatants from each of the patient specimens were used as described above; treatment began 12-13 d after initiation of brain aggregate culture and the specimens were harvested after 1 w.. Brain aggregates were incubated in media alone, $20 \%$ macrophage supernatant (SU), or $20 \%$ supernatant from patients A, B, C, and D. Supernatants from normal macrophages treated with LPS $\left(2 \mu \mathrm{g} / \mathrm{ml}, 48 \mathrm{~h}, 10^{6}\right.$ cells/ $\mathrm{ml}$ ) were tested in parallel. Two human brains were studied.

Trypan blue and lactate dehydrogenase ( $L D H)$ determinations. Each day after treatment, 10-20 brain aggregates were put in trypan blue for $\sim 1 \mathrm{~min}$ and viewed as a wet mount using light microscopy. Exclusion of trypan blue indicated viable cells. Parallel with this examination, $200 \mu \mathrm{l}$ of supernatant fluid was removed, centrifuged at 2,000 rpm for $10 \mathrm{~min}$ at $4^{\circ} \mathrm{C}$, and $100 \mu \mathrm{l}$ of supernatant was removed for LDH assay using spectrophotometric methodology (18). LDH was used as a biochemical index of neural cell injury.

Electron microscopy. Approximately 100 treated and untreated aggregates were sampled after 1 wk and fixed in Karnovsky solution overnight. After fixation, aggregates were placed in cacodylate buffer until further processing. Aggregates were postfixed in $1 \%$ osmium tetroxide dehydrated in 2'2-dimethoxypropane and embedded in polybred 8/2. Thin sections were stained with uranyl acetate and lead citrate and cells within 10 aggregates per experimental condition were examined in a JEOL 100 SX electron microscope.

Histology and immunocytochemistry. Approximately 100 brain aggregates were removed weekly and fixed for $18-24 \mathrm{~h}$ in $2 \%$ paraformaldehyde, washed with PBS, stained with eosin, and embedded in $1 \%$ low temperature melting agarose followed by paraffin. Serial sections $(6 \mu \mathrm{m}$ thick) were stained with hematoxylin and eosin (H \& E), or were used in an indirect avidin biotin immunoperoxidase technique from Vector Laboratories (Burlingame, CA). Astrocytes were identified by antibodies to glial fibrillary acidic protein (GFAP) purchased from Dako Corp. (Santa Barbara, CA) diluted 1:80 and neurons by antibodies to $\alpha$-tubulin diluted 1:80 and purchased from Immunobiologicals (Lisle, IL). Diaminobenzidine from Sigma Chemical Co. (St. Louis, MO) was used 
as the chromogen substrate and sections were counterstained with hematoxylin.

Brain aggregate diameters were determined on 20 aggregates from three different brain specimens held for 3 wk in SU or SI. Brain aggregate diameters in microns were determined on $\mathrm{H} \& \mathrm{E}$ stained slides using a Leitz TAS Image Analyzer at the Laboratory for Cell Analysis (University of California, San Francisco, CA). Statistics were done using the standard Student $t$-test.

Protein, CNP, and neurotransmitter amino acid (NAA) Assays. Flasks containing several thousand aggregates were harvested after 1 wk of culture. Aggregates were solubilized in water, sonicated, and the lysate was aliquoted for protein, CNP, and NAA analyses. Protein was assayed spectrophotometrically in duplicate according to a Micro BCA procedure (Pierce Chemical Co., Rockford, IL).

CNP (19) activity was assayed in duplicate on whole cell lysates after protein determination. Approximately $300 \mu \mathrm{g}$ protein was added to the substrate $2^{\prime}, 3^{\prime}$-cAMP (Sigma Chemical Co.) and allowed to react for $30 \mathrm{~min}$. Alkaline phosphatase was added to hydrolyze the reaction product 2'-AMP. The liberated inorganic phosphate was combined with ammonium molybdate to generate a molybdic acid complex which was measured spectrophotometrically. $1 \mathrm{U}$ of enzyme activity was expressed as nanomoles substrate converted to product per minute of incubation at $30^{\circ} \mathrm{C}$ per milligram protein. Statistics were done using analysis of variance.

NAA levels were analyzed in duplicate at the Protein Structure Core Facility (University of California, Davis, CA) and determined on whole cell lysates by using HPLC. A ninhydrin-based analyzer (model 6300; Beckman Instruments, Fullerton, CA) was used and concentrations were expressed as nanomole/milligram protein. Data for CNP and NAA were initially analyzed by a standard analysis of variance. Paired comparisons between means utilized Newman-Keuls methodology.

Western blot analysis. Western blot analysis using sheep anti-HIV gp120 polyclonal antiserum (courtesy of Dr. John Mills, San Francisco General Hospital, San Francisco, CA) and control sheep serum was performed on equal volumes of supernatant from both HIV-infected T cells as well as HIV-infected macrophages after SDS-PAGE and electroblot transfer to nitrocellulose. An Immunetics (Cambridge, MA) Microblotter 28 was used in a standard Western blot protocol using immune and control sheep serum at a concentration of 1:100 to detect HIV gp120. Biotinylated rabbit anti-sheep IgG (Zymed Laboratories, South San Francisco, CA) was used as a second stage antibody and avidin conjugated to horseradish peroxidase with 4-chloronapathol used as a chromogen for the final step to visualize the Western blot bands.

\section{Results}

In vitro infected macrophages produce factors toxic to human brain cells. All brains exposed to supernatants from HIV-infected macrophages showed similar morphologic changes. No changes from control aggregates were noted in any cultures containing uninfected macrophage supernatant (SU) or supernatant from HIV-infected T cells (Fig. $1 a$ ). Five of seven treated (SI) brain cultures contained aggregates with profound disruption of cellular morphology manifested as peripheral rarefaction or spongiosis after $1 \mathrm{wk}$ in SI (Fig. 1 b). From 20 to $90 \%$ of the aggregates from these brains were affected. In two of three brain aggregate preparations that were cultivated for 3 wk, the spherical diameters in microns of aggregates cultured in SI were significantly smaller than those cultured in SU $(P$ $<0.001$ ). (SU-1 mean $458 \pm 135$, SI-1 $194 \pm 52$; SU-2 mean $411 \pm 132$, SI-2 $204 \pm 66$, mean \pm SD with 20 aggregates measured/culture.)

To test whether the toxic effects observed in Fig. 1 would be specific for a subtype of cell, all brain aggregates were analyzed
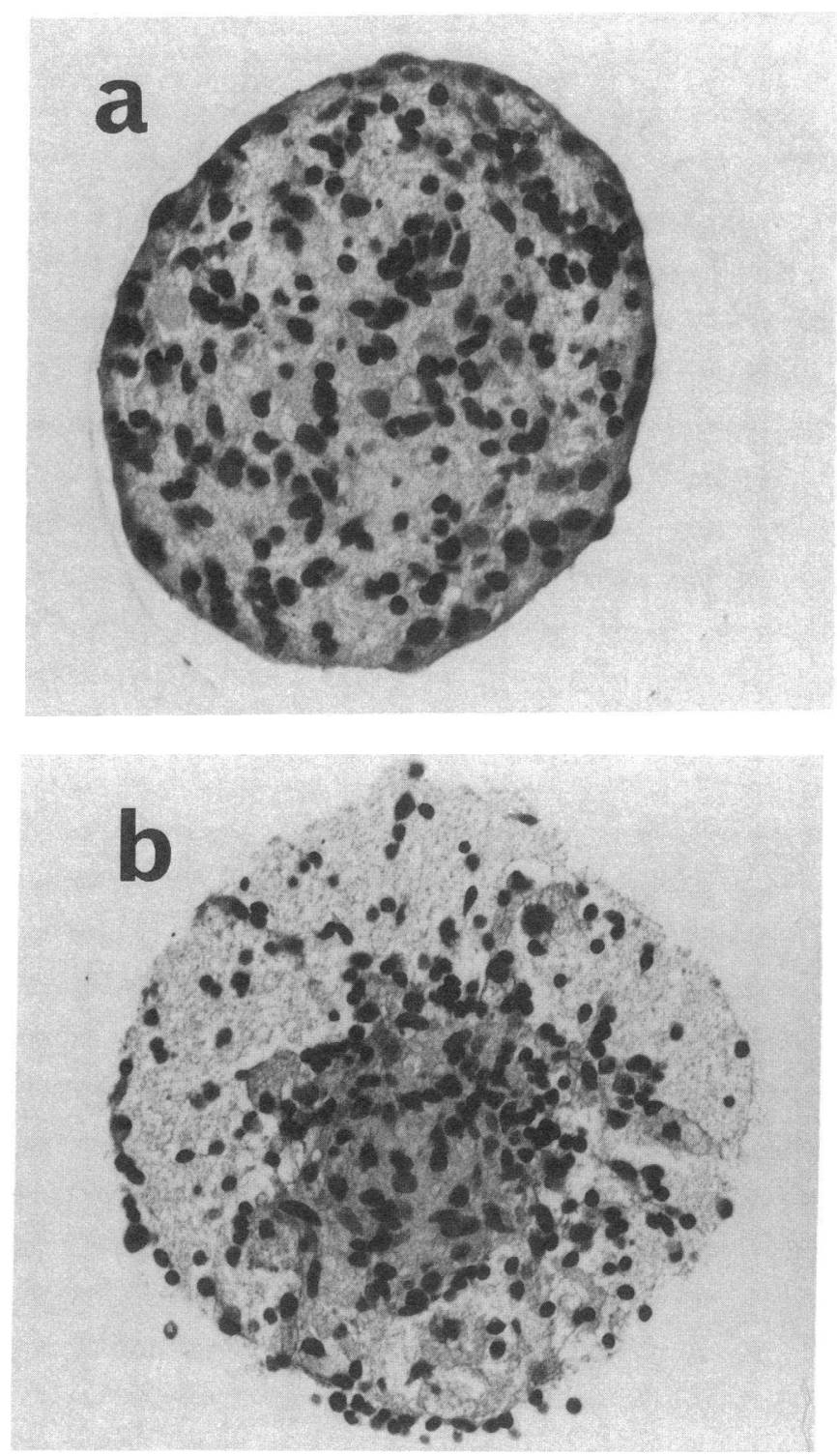

Figure 1. (a) Brain aggregates treated with uninfected macrophage supernatant. H \& E. $\times 100$. (b) Brain aggregates treated with HIVinfected macrophage supernatant for $1 \mathrm{wk}$. Note peripheral rarefaction (arrow). H \& E. $\times 100$. The percentage of aggregates affected from each brain sample treated with in vitro infected macrophage supernatants: brain 1, 90\%; brain 2, 20\%; brain 3, 50\%; brain $4,55 \%$; brain $5,40 \%$; brain $6,20 \%$; brain $7,70 \%$.

for neuronal and astrocytic markers as compared with controls. Fig. 2 shows side-by-side comparison of SI exposed aggregates (Fig. $2 c, d$ ) as compared with control aggregates (Fig. $2 a$, $b$ ). Using this method of analysis, no specific cellular subtype appeared most sensitive to SI-mediated toxicity although two of seven brain aggregates showed a decrease in neurons (Fig. 2 $b, d)$. By electron microscopic ultrastructural examination, the peripheral cells within aggregates were unrecognizable as to cellular type and contained multiple cytoplasmic vacuoles. Cells recognizable as neurons, astrocytes, or oligodendrocytes also contained cytoplasmic vacuoles. Fig. 3 demonstrates the ultrastructural differences between normal (Fig. $3 a$ ) as compared with SI exposed brain aggregates (Fig. $3 b$ ). 

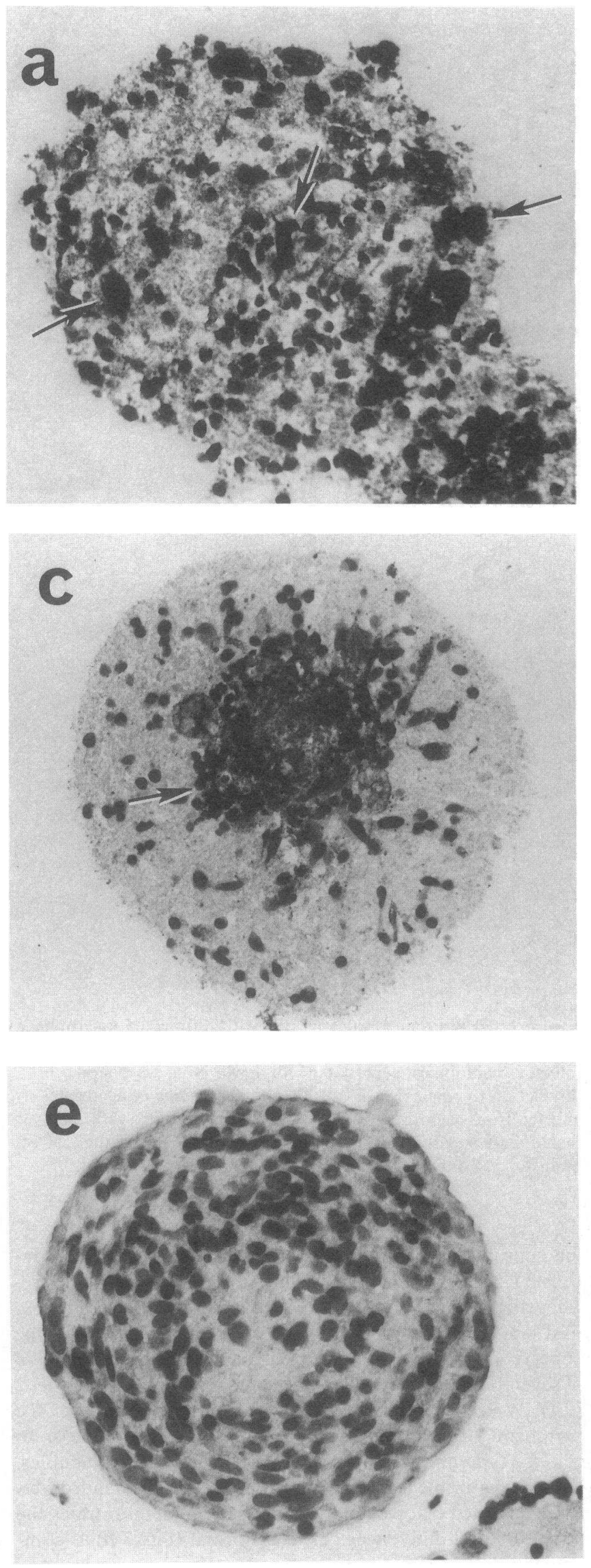
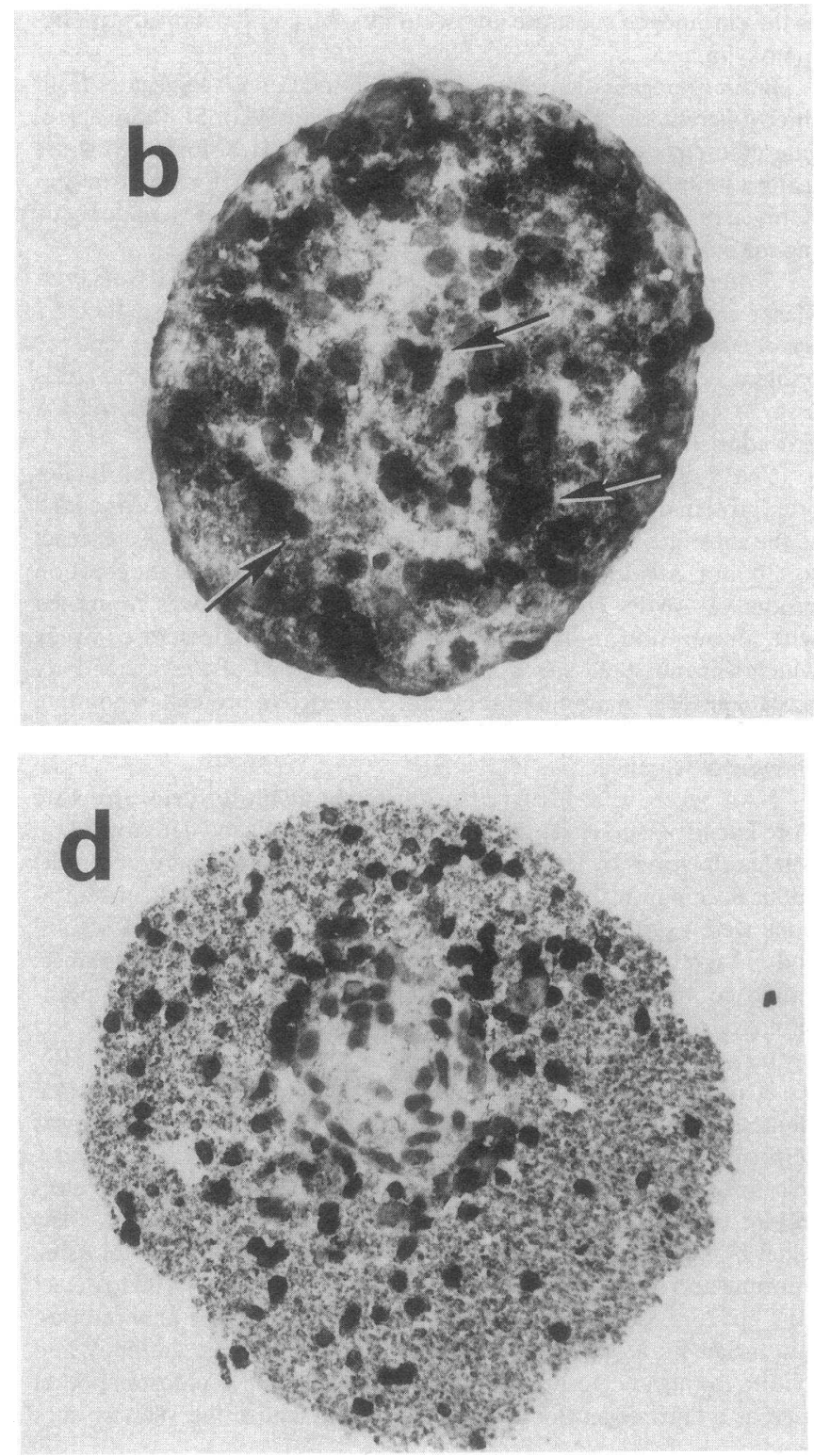

Figure 2. Brain aggregates treated with uninfected macrophage supernatant ( $a$ and $b$ ) or HIV-infected macrophage supernatant ( $c$ and $d$ ) and stained immunocytochemically with antibody to glial fibrillary acidic protein (GFAP) $(a$ and $c$ ) or $\alpha$-tubulin ( $b$ and $d$ ). Arrows indicate positively staining cells that were seen throughout the aggregates treated with uninfected macrophage supernatant. Brain aggregates treated with HIV-infected macrophage supernatant and stained with anti- $\alpha$-tubulin showed a diffuse peripheral staining pattern with no cells staining positive $(d)$ and the central cells staining with GFAP $(c)$. Brain aggregate controls included staining immunocytochemically with normal rabbit serum $(e)$. A diaminobenzidine substrate was used and the aggregates were counterstained with hematoxylin. $\times 100$. 

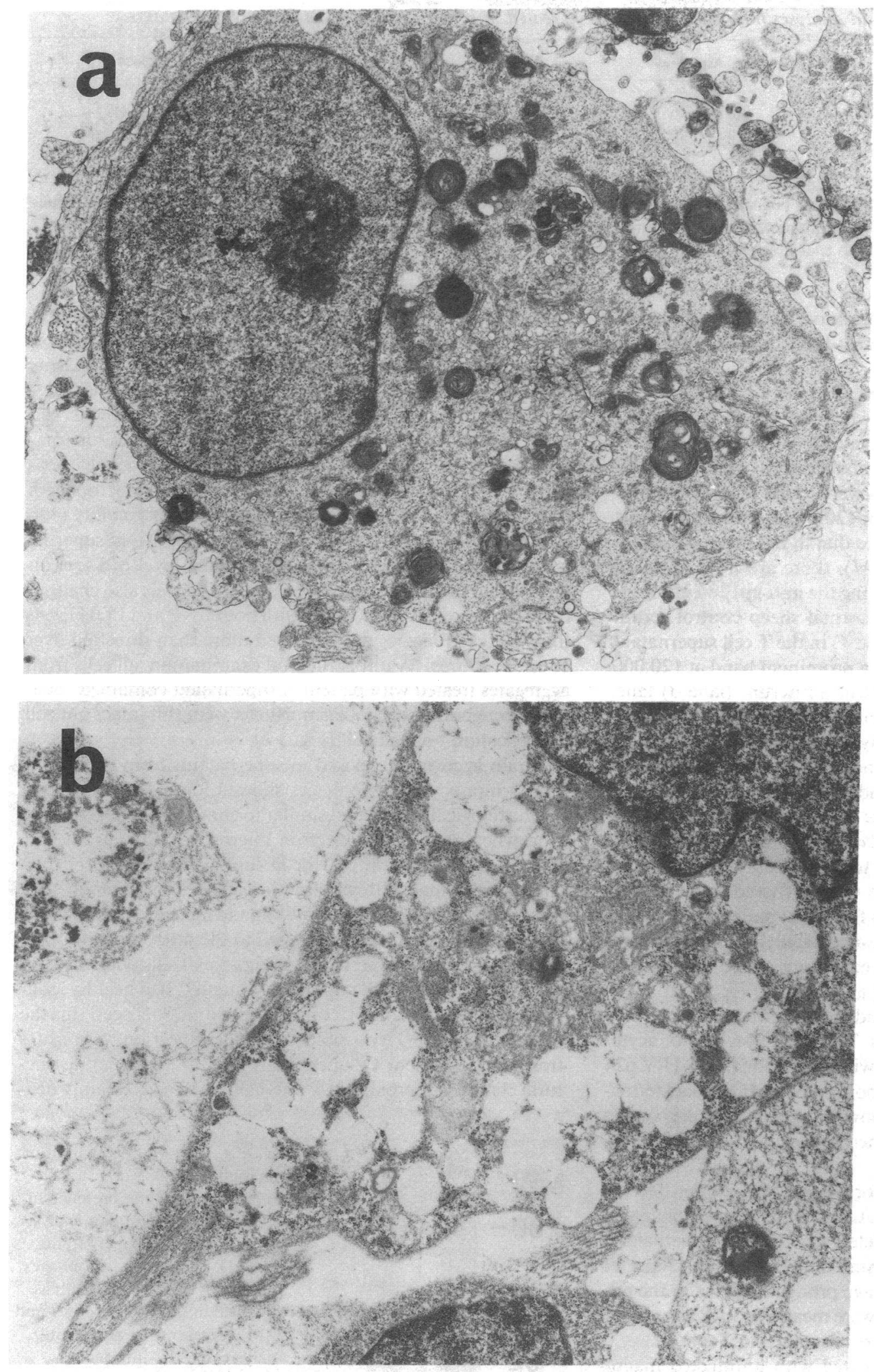

Figure 3. (a) Electron micrograph of a normal neuron with a prominent nucleolus from an untreated brain aggregate. The cytoplasm contains numerous organelles including myelin. $\times 9,765$. (b) Electron micrograph of an astrocyte with a process containing filaments from aggregates treated with HIV-infected macrophage supernatant. Note numerous vacuoles within the cytoplasm. $\times 11,960$.

Excitatory and inhibitory NAAs were measured as compared with the structural amino acid leucine in four brains to test whether neuronal function would be altered by SI. All five neurotransmitter amino acid levels were depressed in the SI exposed brain aggregates relative to controls (Table I). How- ever, statistical differences between the individual amino acids and their respective controls could not be demonstrated ( $P$ $>0.05$ ). CNP levels were measured in SI-exposed brain aggregates and appropriate controls for seven brain preparations (24 control samples, 30 treatment samples). The difference was not 
Table I. Neurotransmitter Amino Acid Analyses from Brain Aggregates Treated with HIV-infected Macrophage Supernatants

\begin{tabular}{lccccc}
\hline & Taurine & Asp & Glu & Gly & Gaba \\
\hline Control & $2.83 \pm 1.29$ & $0.87 \pm 0.36$ & $2.50 \pm 1.48$ & $3.41 \pm 0.80$ & $2.32 \pm 1.97$ \\
1 wk & $2.46 \pm 1.49$ & $0.84 \pm 0.30$ & $2.18 \pm 0.89$ & $2.93 \pm 0.41$ & $1.98 \pm 1.45$
\end{tabular}

Data were collected on four brain preparations. NAA levels were normalized to the level of leucine present in the brain aggregate with the value of leucine equal to 1.00 . Before normalization, measurements of amino acids were expressed as nanomoles/milligram of protein. Each brain preparation consisted of at least two trials. No significant difference $(P>0.05)$ could be demonstrated for an individual AA.

statistically significant. The cumulative normalized results were as follows: control; $1.00 \pm 0.3 \mathrm{SI} ; 1: 10 \pm 0.24, P=0.3$, Student's $t$ test.

HIV gp120 has been reported to cause toxic changes in rodent neural cells (10). To test whether the toxic effects observed in SI-exposed brain aggregates would be mediated by HIV gp120, equal volumes of the macrophage supernatant and HIV-infected $T$ cell supernatants were tested by Western blot analysis for the presence of HIV gp120. Fig. 4 shows the results of this Western blot analysis. Note that in the macrophage supernatant Western blot lanes $(M)$, there are no significant bands present in the lane containing the anti-gp120 antiserum (lane 2) as compared with the normal sheep control serum (lane 1). The anti-gp120 lane (lane 4) in the T cell supernatant Western blots $(T)$, however, had a prominent band at 120,000 mol wt, not present in the control antiserum (lane 3) lane. These results show that the level of gp1 20 present in macrophage supernatants is apparently substantially lower than in the $T$ cell supernatants. These findings make the findings seen in Figs. 1-3 unlikely to be secondary to effects of HIV gp120.

Monocyte/macrophages from AIDS patients produce factors toxic to human brain cells. To test whether in vivo derived macrophages would produce factors toxic to human brain cell aggregates, culture supernatants were prepared from PBMCderived monocyte/macrophages from four patients with AIDS (unlinked; no clinical information available). Monocyte/macrophages isolated by glass adherence were placed into suspension culture at $5 \times 10^{5}$ cells $/ \mathrm{ml}$ and cultured for $5 \mathrm{~d}$ at $37^{\circ} \mathrm{C}$. These cells were centrifuged and the supernatants were processed as described in Methods. The 5-d cultured monocyte/ macrophages were then stained with monoclonal anti-HIV p24 and control MAbs and the proportion of $\mathrm{p} 24^{+}$monocyte/macrophages was quantitated by flow cytometry. The proportion of $\mathrm{p} 24^{+}$monocytes at day 5 varied between $<1.5$ to $8.8 \%$ (Table II).

To test whether toxic factors existed in patient-derived monocyte/macrophage supernatants, patient macrophages as compared with control uninfected macrophage supernatants were added to brain aggregates similar to experiments reported with in vitro infected macrophage produced SI. As in the earlier studies, several parameters were measured $1 \mathrm{wk}$ after initiation of culture, and results are summarized in Table II.

Brain aggregate cultures treated with uninfected macrophage supernatants or HIV-infected T cell supernatants had recognizable neurons, astrocytes, and oligodendrocytes. Brain aggregates treated with supernatants from patients A and B histologically and by ultrastructural examination had a similar appearance to aggregates treated with SI.

Supernatant from patient B $\left(8.8 \% \mathrm{p} 24^{+}\right.$cells $)$caused the most profound abnormalities in cultured brain aggregates.
Trypan blue staining of patient B treated aggregates showed virtually $100 \%$ cell death confirmed by transmission electron microscopy (Fig. 5 a) (100 cells from each). Cell cytoplasms were lacking organelles and the nuclei appeared as shells devoid of chromatin. Morphologic changes consistent with cell death were accompanied by elevated levels of LDH in supernatants from patient B's culture (Table II). Brain aggregates exposed to supernatants from patient A monocyte/macrophages ( $7.1 \% \mathrm{p} 24^{+}$cells) also showed decreased cellular viability (30$40 \%$ dead cells assessed by trypan blue staining) and supernatants showed a correspondingly increased level of LDH although less elevated than in patient B. CNP levels also changed in relation to observed toxicity with both CNP and LDH levels from patient $B$ aggregates increased more than threefold over control cultures. By ultrastructural examination, all cells from aggregates treated with patient A supernatant contained vacuoles throughout the cytoplasm. Nuclei were still intact but cell identification was difficult (Fig. $5 b$ ).

Brain aggregates exposed to supernatants from patients $C$ and D monocyte/macrophages showed lower levels of LDH and histologically appeared similar to control aggregates. However, when analyzed by electron microscopy all neurons and astrocytes treated with patient $D$ supernatant (Fig. $5 c$ ) contained more than 10 cytoplasmic vacuoles. Neurons within these aggregates also showed a dramatic increase in mitochondria not seen in aggregates exposed to the other patient supernatants or culture supernatants (Fig. $5 c$ ). Cells within aggregates exposed to supernatants from patient $C$ had healthy looking nuclei and normal organelles could be seen in the cytoplasm, making it possible for cell identification. Aggregates treated with patient $\mathrm{C}$ supernatant had the fewest ultrastructural changes: approximately one-half of the cells (mainly neu-

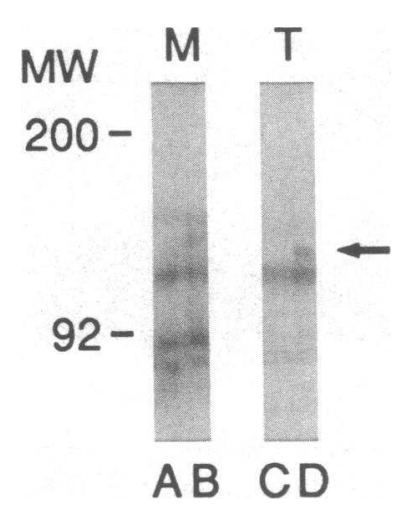

Figure 4. Western blot analysis of macrophage and $T$ cell supernatants for the presence of HIV gp120. Western blot analysis was performed on supernatants from HIV-infected macrophages as well as HIV-infected T cells as described in Methods. Lanes 1 and 2 (M) represent Western blot analysis on HIV-infected macrophage supernatants with (lane 1 ) normal sheep serum and (lane 2) anti-gp120 sheep antiserum. Lanes 3 and 4 represent Western blot analysis on HIV-

infected $\mathrm{T}$ cell supernatants with lane 3 (normal sheep serum) and lane 4 (the anti-gp 120 sheep serum). The arrow on the right indicates gp120 reactivity present only in lane 4. 
Table II. Brain Aggregate Cultures Exposed to Control and Patient Macrophage Supernatants

\begin{tabular}{lcccr}
\hline & $n^{*}$ & $\mathrm{p}^{4} 4^{*}$ & CNP' & LDH $^{\mathbf{1}}$ \\
\hline & & $\%$ & & \\
Control (brain 1) & 2 & 0 & 1.00 (normalized) & 270 \\
Patient A & 4 & 7.1 & $1.65 \pm 0.07$ & 930 \\
Patient B & 4 & 8.8 & $3.05 \pm 0.17$ & 1514 \\
Control (brain 2) & 2 & 0 & 1.00 (normalized) & 333 \\
LPS' & 2 & 0 & $1.22 \pm 0.02$ & 418 \\
Patient C & 2 & $<1.5$ & $1.66 \pm 0.07$ & 331 \\
Patient D & 2 & 5.7 & $2.25 \pm 0.11$ & 329 \\
& & & &
\end{tabular}

* $n$ Represents the number of samples derived from a particular brain (brain 1: control, A, B; and brain 2: control, LPS, C, D).

${ }^{\text {}}$ Percent of patient monocyte/macrophages expressing HIV p24 above background.

${ }^{8}$ The LPS and patient supernatants caused significantly greater brain aggregate $\mathrm{CNP}$ levels when compared with their respective controls (Student's $t$-test, $P<0.05$ ).

I LDH (lactate dehydrogenase) activity given in units/milliliter of brain aggregate supernatant.

' LPS stimulated ( $2 \mu \mathrm{g} / \mathrm{ml}$ for $48 \mathrm{~h}$ ) macrophage supernatant.

rons and astrocytes) contained some cytoplasmic vacuolation (Fig. $5 d$ ).

To test whether the observed toxicities mediated by SI in brain aggregate cultures would be produced by factors elaborated merely by activated macrophages, supernatant from macrophages activated by LPS was also tested in parallel with patient C and D's specimen. Table II shows that both CNP and LDH levels were increased relative to control supernatants; however, ultrastructurally the brain aggregates exposed to activated macrophage supernatants were unchanged from control aggregates with no cytoplasmic vacuolation detected in either specimen. The overall meaning of increased levels of CNP induced by the patient specimens as well as LPS activated supernatant is unclear as electron microscopic examination of those brains failed to show increased levels of myelin. Similarly the meaning of elevated LDH levels in patient B exposed aggregates almost certainly related to cell death as confirmed by electron microscopy (Fig. $5 a$ ).

Ultrastructural changes occurring in brain aggregates appeared to most closely correlate with the proportion of macrophages expressing HIV p24. Note that the brain aggregates exposed to supernatant from patient $C$ show very little ultrastructural damage; however, there is progressive change from patients D to A to B with increased evidence of toxicity occurring as the proportion of $\mathrm{p} 24^{+}$cells increased. These data suggest that factors produced by HIV-infected macrophages derived from AIDS patients were capable of causing severe ultrastructural changes, changes that did not occur when parallel brain aggregates were exposed to LPS-activated macrophages known to produce high levels of IL- 1 and TNF- $\alpha$. Factors responsible for these ultrastructural changes have not yet been characterized.

\section{Discussion}

In this paper we describe an in vitro culture system that may be a model for AIDS-associated dementia. Supernatants derived from HIV-infected macrophages caused ultrastructural and functional changes in human brain aggregates cultured in vitro. The observed changes were specific for brain aggregates exposed to supernatants from infected macrophages, as HIVinfected $T$ cell supernatants, uninfected and LPS-stimulated macrophage supernatants did not cause any measurable changes in the brain aggregates. The effects were caused by supernatants derived from macrophages infected in vitro as well as macrophages isolated from patients with AIDS.

The principle and most reproducible findings in brain aggregates exposed to HIV-infected macrophage supernatants were toxic changes in all neural cell types. Immunocytochemical analysis of control as compared with SI-exposed brain aggregates found that no specific subtype was preferentially depleted in SI-exposed cultures except in two of nine brain aggregates where there was a prominent loss of neurons. The histochemical hallmarks of SI-exposed brain aggregates were rarefaction and spongiform changes, changes that occurred in 20-90\% of aggregates in SI-exposed cultures. Ultrastructurally, these features appeared as vacuolar changes in cytoplasms of the SI-exposed cells. The most profound change was cellular death as manifested by organelle dropout, loss of cellular integrity with parallel increases in supernatant LDH.

Supernatants from high virus-expressing (8.8\% p24) AIDS patient-derived macrophages caused the most cellular destruction in brain aggregates. The widespread histochemical and ultrastructural damage was confirmed by dramatic trypan blue incorporation and high LDH release. Brain aggregates exposed to supernatants from the other three patients $(A, C, D)$ showed a decreasing severity of toxic changes correlating with the degree of macrophage HIV expression. Patient A supernatants showed both cytoplasmic vacuolation in all cells as well as clumping of nuclear chromatin by electron microscopic analysis, coupled with intermediately elevated levels of supernatant LDH. Only subtle changes in cellular vacuolation $(\sim 50 \%$ of neurons) were present in the brain aggregates exposed to patient $\mathrm{C}$ supernatant $\left(<1.5 \% \mathrm{HIV}^{+}\right.$monocytes).

One of the differences between brain aggregates exposed to supernatants from in vitro infected macrophages as compared with the patient-derived specimens, were results of CNP measurements. Collectively, the seven brains exposed to in vitro infected macrophage supernatants showed moderate but not statistically significant increases relative to controls in brain aggregate CNP activity. Brain aggregates exposed to patient specimens all showed increased levels of CNP, without ultrastructural evidence for increases in oligodendrocyte numbers or myelin. The major variables that might account for these disparate results likely revolve around the macrophage supernatant sources. Patient-derived macrophages are infected with native or "wild-type" HIV, which may function within macrophages in a very different manner than the in vitro propagated HIV $-1_{D V}$ strain of virus. Another variable may be related to degree of macrophage activation and/or differentiation between the two supernatant sources. The patient specimens were cultured for only $5 \mathrm{~d}$ after glass activation whereas the in vitro infected macrophages had been in suspension culture for almost $4 \mathrm{wk}$, including $2 \mathrm{wk}$ after in vitro infection. The patient specimens would represent HIV-infected macrophages substantially more activated than the in vitro infected cells; however, macrophage activation alone (with LPS) was not sufficient to cause the toxic effects observed in the exposed brain aggregates. Further studies of these two macrophage sources 

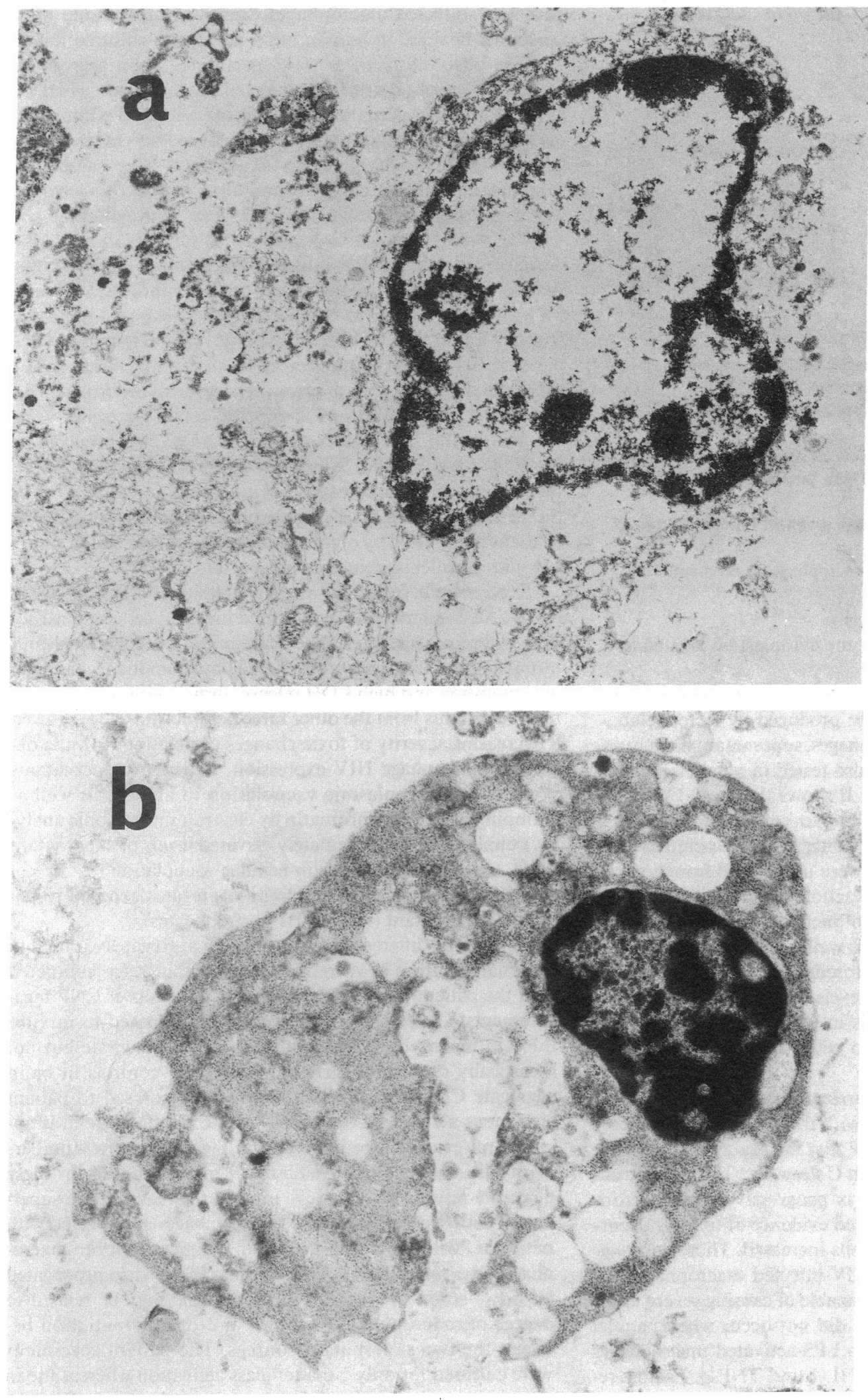

Figure 5. 

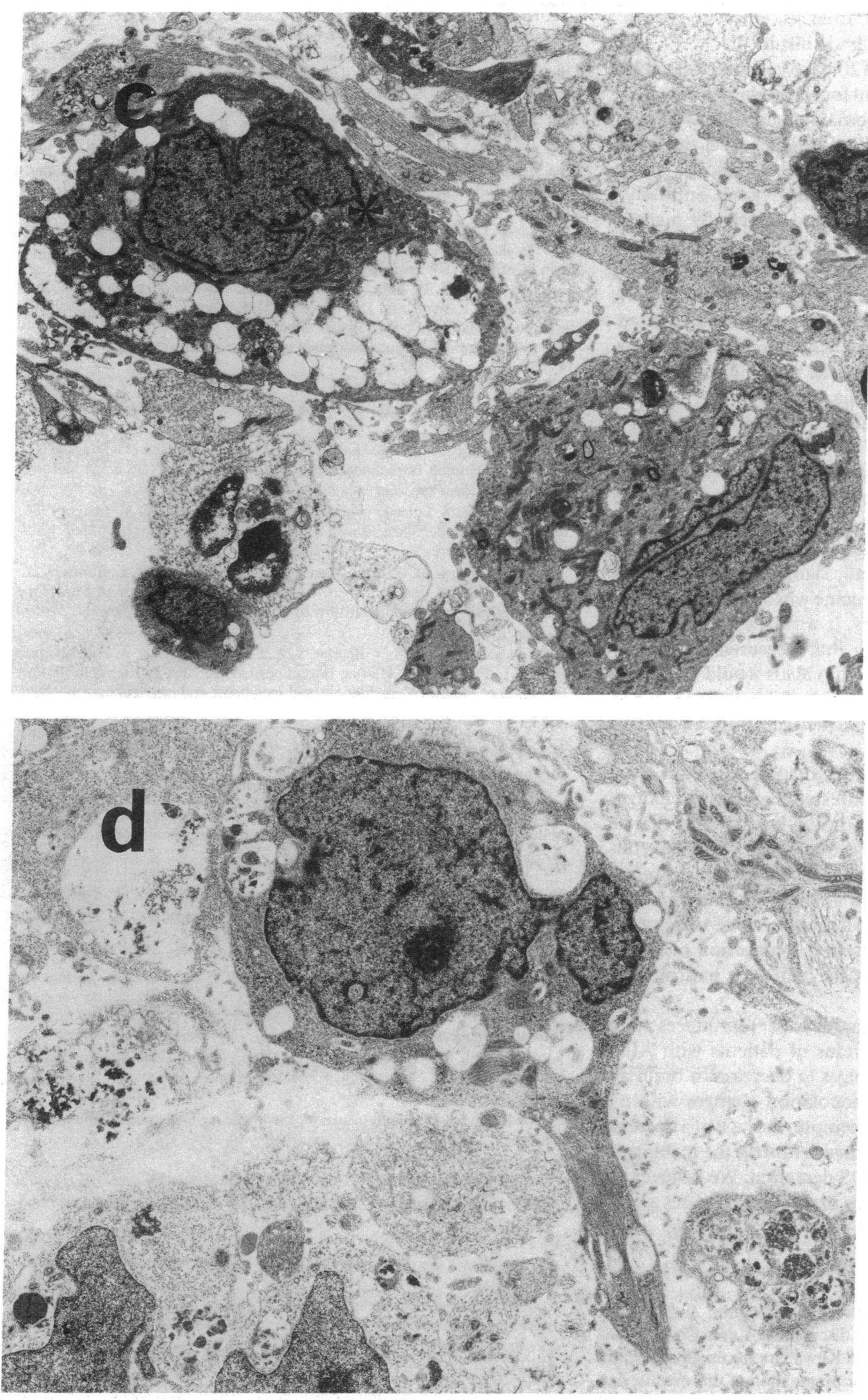

Figure 5. Transmission electron micrographs of brain aggregate cells exposed to patient macrophage supernatants. Electron micrographs $a-d$ are representative of the morphology of the major cellular phenotype present in patient supernatant exposed aggregates after 100 cells/specimen were examined. (a) Electron micrograph of a cell from a brain aggregate treated with supernatant from patient $B$ (8.8\% p2 $4^{+}$macrophages). All cells showed final stages of cell death. Cytoplasms were lacking organelles and nuclei were devoid of chromatin. $\times 9,675$. (b) Electron micrograph of an unidentified cell from a brain aggregate treated with supernatant from patient $A$

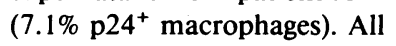
cells contained vacuoles within the cytoplasm and clumped chromatin within the nucleus. $\times 14,175$. (c) Electron micrograph of two neurons from aggregates treated with supernatant from patient $D$ (5.7\% p24 $4^{+}$macrophages).

Numerous vacuoles appeared in the cytoplasm with an increase in the number of mitochondria ${ }^{*}$ ), as compared with neurons observed in control aggregates. $\times 4,805$. (d) Electron micrograph of an astrocyte with a process containing filaments from a brain aggregate treated with supernatant from patient $C$ $\left(<1.5 \%{\mathrm{p} 24^{+}}^{+}\right.$macrophages). The cytoplasm contained more vacuoles than cells in control aggregates although the nucleus appeared normal. $\times 7,955$. will be required to identify factors that resulted in the CNP activity increases.

The increase in CNP activity from in vivo infected macrophages may have been secondary to a stress response, and may suggest active attempts at repair of damage; increased mito- chondrial activity was seen in neurons containing a moderate degree of vacuolation (Fig. 5 c). In other systems, CNP levels are known to increase in response to cyclic AMP and its inducers such as norepinephrine and prostaglandin $\mathrm{E}_{1}(20,21)$ and growth factors such as somatomedin C (22). It is possible 
that the increased CNP activity occurred secondary to growth factor elaboration in response to a toxic insult.

Virus gene products such as gp 120 have been implicated in some in vitro experiments to account for HIV-related neurotoxicity (10). However, our T cell supernatant shown by Western blot analysis to be secreting gp120 at a higher level than the infected macrophage cultures did not cause significant structural and functional changes in the brain aggregates. Similarly, the toxic effects are likely not elaborated merely by macrophage activation, as LPS-activated macrophage supernatants caused no ultrastructural changes in exposed brain aggregates. There were only slight increases in CNP activity and LDH levels in the LPS-activated macrophage supernatant exposed brain aggregates. Therefore, it is unlikely that a factor such as TNF- $\alpha$ was responsible for mediating the toxic effects observed in this study, and in a parallel analysis, no TNF- $\alpha$ was found in supernatants from in vitro HIV-infected macrophages.

Fluctuations in inhibitory and excitatory neurotransmitter amino acids were assessed to determine their potential fole in soluble factor induced brain dysfunction. Recent reports have shown that excitatory amino acids (EAA) can cause secondary brain tissue injury (23). All five neurotransmitter amino acid levels showed a slight, not statistically significant decrease relative to the structural amino acid leucine when exposed to HIVinfected macrophage supernatants. It was believed, based on some preliminary data (not shown), that measurements of subtle changes in neurotransmitter amino acids would not be interpretable in the setting of widespread cellular damage seen in brain aggregates exposed to patient derived infected macrophages.

All of the brain aggregates exposed to supernatant from AIDS patient macrophages manifested significant changes both morphologically and enzymatically. It is unlikely that all four patients had AIDS-associated dementia. Therefore, further analysis in a controlled fashion of monocytes from patients with AIDS dementia as well as those without dementia will be required in order to test whether levels of toxicity observed in aggregates are related to both proportion of monocytes expressing HIV as well as the patient's neurological status.

The in vitro model described in this paper reproduces many of the abnormalities found in brains of patients with AIDS dementia including atrophic changes (a decrease in brain aggregate size), rarefaction, and vacuolation changes within a wide spectrum of cell types. Furthermore, the lack of a decrease in CNP activity in the model is consistent with the inability to correlate demyelination with AIDS dementia. We believe that the culture system described in this paper approximates a valid model for in vitro study of AIDS-associated dementia.

\section{Acknowledgments}

We thank Ray Swanson for LDH determinations; John Ziegler, Scott Panther, and Linda Noble for helpful discussions; Karen Chew, Diane George, Isabelle Gaston, and Thomas Kuwahara for excellent technical assistance; and John Flickinger for preparation of this manuscript.

This work was supported in part by the California Universitywide AIDS Taskforce and the Veterans Administration Center for AIDS Research and Education.

\section{References}

1. Price, R. W., and B. J. Brew. 1988. The AIDS dementia complex. J. Infect. Dis. 158:1079-1083.
2. Navia, B. A., E. S. Cho, C. K. Petito, and R. W. Price. 1986. The AIDS dementia complex: II Neuropathology. Ann. Neurol. 19:525-535.

3. Vazeux, R., N. Brousse, A. Jarry, D. Henin, C. Marche, C. Vendrenne, J. Mikol, M. Wolff, C. Michon, W. Rozenbaum, J.-F. Bureau, L. Montagnier, and M. Brahic. 1987. AIDS subacute encephalitis. Identification of HIV-infected cells. Am. J. Pathol. 126:403-410.

4. Shaw, G. M., M. E. Harper, B. H. Hahn, L. G. Epstein, D. C. Gajdusek, R. W. Price, B. A. Navia, C,K. Petito, C. J. O'Hara, J. E. Groopman, E. S. Cho, J. M. Leske, F. Wong-Staal, and R. C. Gallo. 1985. HTLV-III infection in brains of children and adults with AIDS encephalopathy. Science (Wash. DC). 227:177180.

5. Levy, J. A., J. Shimabukuro, H. Hollander, J. Mills, and L. Kaminsky. 1985. Isolation of AIDS-associated retroviruses from cerebrospinal fluid and brain of patients with neurological symptoms. Lancet. ii:586-588.

6. Ho, D. D., T. R. Rota, R. T. Schooley, J. C. Kaplan, J. D. Allan, J. E. Groopman, L. Resnick, and D. Felsenstein. 1985. Isolation of HTLV-III from cerebrospinal fluid and neural tissues of patients with neurologic syndromes related to the acquired immunodeficiency syndrome. N. Engl. J. Med. 313:14931497.

7. Hollander, H., and J. A. Levy. 1987. Neurologic abnormalities and recovery of human immunodeficiency virus from cerebrospinal fluid. Ann. Intern. Med. 106:692-695.

8. Cheng-Mayer, C., J. Rutka, M. Rosenblum, C. McHugh, D. Stites, and J. Levy. 1987. Human immunodeficiency virus can productively infect cultured human glial cells. Proc. Natl. Acad. Sci. USA. 84:3526-3530.

9. Chiodi, F. S. Fuerstenberg, M. Gidlund, B. Asjo, and E. M. Fenyo. 1986 Infection of brain-derived cells with the human immunodeficiency virus. J. Virol. 61:1244-1247.

10. Brenneman, D. E., G. L. Westbrook, S. F. Fitzgerald, D. L. Ennist, K. L. Elkins, M. R. Ruff, and C. B. Pert. 1988. Neuronal cell killing by the envelope protein of HIV and its prevention by vasoactive intestinal peptide. Nature (Lond.). 335:639-642.

11. Wahl, L. M., M. L. Corcoran, S. W. Pyle, L. O. Arthur, A. Harel-Bellan, and W. L. Farrar. 1989. Human immunodeficiency virus glycoprotein (gp120) induction of monocyte arachidonic acid metabolite and interleukin. Proc. Natl. Acad. Sci. USA. 86:621-625.

12. Gurney, M. E., S. P. Heinrich, M. R. Lee, and H.-S. Yin. 1986. Molecular cloning and expression of neuroleukin, a neurotropic factor for spinal and sensory neurons. Science (Wash. DC). 23:566-574.

13. Crowe, S., J. Mills, and M. S. McGrath. 1987. Quantitative immunocytofluorographic analysis of CD4 surface antigen expression and HIV infection of human peripheral blood monocyte/macrophages. AIDS Res. Hum. Retroviruses. 3:135-145.

14. McGrath, M. S., K. M. Hwang, S. E. Caldwell, I. Gaston, K.-C. Luk, P. Wu, V. L. Ng, S. Crowe, J. Marsh, T. Deinhart, P. V. Lekas, J. C. Vennari, H.-W. Yeung, and J. D. Lifson. 1989. GLQ223: an inhibitor of human immunodeficiency virus replication in acutely and chronically infected cells of lymphocyte and mononuclear phagocyte lineage. Proc. Natl. Acad. Sci. USA. 86:2844-3848.

15. Locksley, R. M., S. Crowe, M. D. Sadick, F. P. Heinzel, K. D. Gardner, Jr., M. S. McGrath, and J. Mills. 1988. Release of interleukin 1 inhibitory activity (contra IL-1) by human monocyte-derived macrophages infected with human immunodeficiency virus in vitro and in vivo. J. Clin. Invest. 82:2097-2105.

16. Pulliam, L., M. E. Berens, and M. L. Rosenblum. 1988. A normal human brain cell aggregate model for neurobiological studies. J. Neurosci. Res. 21:521530.

17. Kurihara, T., and Tsukada, Y. 1967. The regional and subcellular districution of $2^{\prime}, 3^{\prime}$-cyclic nucleotide 3 '-phosphohydrolase in the central nervous system. J. Neurochem. 14:1167-1174.

18. Koh, J. Y., and D. W. Choi. 1987. Quantitative determination of glutamate mediated cortical neuronal injury in cell culture by lactate dehydrogenase efflux assay. J. Neurosci. Res. 20:83-90.

19. Prohaska, J. R., D. A. Clark, and W. W. Wells. 1973. Improved rapidity and precision in the determination of brain 2'3'-cyclic nucleotide $3^{\prime}$-phosphohydrolase. Anal. Biochem. 56:275-282.

20. McMorris, F. A., T. M. Smith, T. J. Sprinkle, and J. S. Auszmann. 1985. Induction of myelin components: cyclic AMP increases the synthesis rate of $23^{\prime}$-cyclic nucleotide $3^{\prime}$-phosphohydrolase in $\mathrm{C} 6$ glioma cells. J. Neurochem. 44:1242-1251.

21. McMorris, F. A. 1983. Cyclic AMP induction of the myelin enzyme $2^{\prime}, 3^{\prime}$ cyclic nucleotide 3 '-phosphohydrolase in rat oligodendrocytes. J. Neurochem. 41:506-515.

22. McMorris, F. A., T. M. Smith, S. Desalvo, and R. W. Furlanetto. 1986. Insulin-like growth factor $\mathrm{l} /$ somatomedin $\mathrm{C}$ : A potent inducer of oligodendrocyte development. Proc. Natl. Acad. Sci. USA. 83:822-826.

23. Faden, A. I., P. Demediuk, S. S. Panter, and R. Vink. 1989. The role of excitatory amino acids and NMDA receptors in traumatic brain injury. Science (Wash. DC). 244:798-800. 